\title{
Concentrated Bone Marrow Aspirate and Subacromial Bursa-Derived Cells Demonstrate Similar Cellular Adhesion and Proliferation Potential on Demineralized Bone Matrix Scaffolds for Biologic Augmentation of Rotator Cuff Repair
}

\author{
L. N. Muench ${ }^{1,2}$, D. P. Berthold ${ }^{1,2}$, C. Kia ${ }^{1}$, A. Lebaschi ${ }^{1}$, M. P. Cote ${ }^{1}$, M. B. McCarthy ${ }^{1}$, \\ A. D. Mazzocca ${ }^{1}$
}

1 Department of Orthopaedic Surgery, UConn Health, Farmington, CT, USA

2 Department of Orthopaedic Sports Medicine, Technical University of Munich, Munich, Germany

\author{
CORRESPONDING AUTHOR: \\ Lukas N. Muench \\ Department of Orthopaedic Surgery \\ UConn Health Center \\ 263 Farmington Ave \\ Farmington CT 06030, USA \\ E-mail: lukas.muench@tum.de \\ Phone: +1 860-679-2640 \\ Fax: +1 860-679-2109 \\ DOI: \\ 10.32098/mltj.01.2020.06
}

LEVEL OF EVIDENCE: Controlled Laboratory Study

\begin{abstract}
SUMMARY
Background. Specific scaffolds are still required to sufficiently deliver and retain applied mesenchymal stem cells (MSCs) of various origins to the targeted zone of repaired tissue. The purpose of the study was to evaluate the demineralized bone matrix (DBM) as a scaffold for concentrated bone marrow aspirate (cBMA), subacromial bursa-derived cells (SBDCs), tenocytes, and osteoblasts.

Methods. cBMA was harvested from the proximal humerus along with tenocytes and subacromial bursal tissue during arthroscopic shoulder surgery. Human osteoblasts were obtained from femoral condyles of patients undergoing total knee arthroplasty. Cells were seeded on a DBM scaffold. Cellular adhesion and proliferation assays were performed for each scaffold sample. Half of cBMA underwent arthroscopic simulation of being washed to mimic the intraoperative environment.

Results. There was no significant difference in cellular adhesion potential on DBM scaffolds $(\mathrm{p}=0.168)$ when comparing SBDCs (mean \pm SD: $25,775 \pm 11,351)$, osteoblasts (mean \pm SD: $33,113 \pm 2,709$ ), tenocytes (mean \pm SD: $25,000 \pm 3,864$ ) and cBMA (mean \pm SD: 25,025 $\pm 6,206$ ). Osteoblasts (mean \pm SD: $0.279 \pm 0.009)$ showed a significantly higher proliferation potential $(\mathrm{p}=0.021$, respectively) compared to SBDCs (mean \pm SD: $0.248 \pm 0.015)$, tenocytes (mean \pm SD: $0.239 \pm 0.011$ ), and cBMA (mean \pm SD: $0.247 \pm 0.007$ ). Histology sections and electron microscopy confirmed the presence of cells within the matrix of scaffolds.

Conclusions. SBDCs and cBMA demonstrated high cellular adhesion and proliferation potential on DBM scaffolds.
\end{abstract}

\section{KEY WORDS}

Biologic augmentation; concentrated bone marrow aspirate; demineralized bone matrix; rotator cuff repair; scaffold; shoulder surgery; subacromial bursa

\section{BACKGROUND}

As re-tears following rotator cuff repair remain a major challenge in orthopaedic surgery, the use of biologic adjuvants has garnered recent attention in an attempt to improve healing potential $(1,2)$ Clinical application of autologous mesenchymal stem cells (MSCs) has been shown to be a viable option to create an improved healing environment (2-7). Bone marrow is considered the most common source for MSCs, as it can easily be harvested from the proximal humerus or iliac crest during surgery, and be re-applied to the tear site following concentration $(2,3,5,8)$. In addition to bone marrow, subacromial bursa has recently been proposed as 
an easily accessible alternative source for MSCs, highlighting its potential as a biologic augment in the setting of rotator cuff surgery (9-11).

Despite these improvements in biologic therapies, extracellular matrix of specific scaffolds is still required to sufficiently deliver and retain applied MSCs to the targeted zone of repaired tissue (12). As artificial scaffolds require biocompatibility in order to ensure retaining the seeded MSCs and mimic natural properties, repair augmentation using demineralized bone matrix (DBM) scaffolds has recently emerged (12-16). DBM scaffolds are a composite of collagen, several growth factors, along with calcium phosphate. Its spongy structure allows for flexibility in the hydrated state, while maintaining an osteoinductive potential $(12,13,16)$. In animal models, DBM has been shown to improve tendon-bone healing and to restore bone mineral density at the enthesis, highlighting its potential role as an augment in rotator cuff healing (14-16). In addition, Hoberman et al. demonstrated that DBM scaffolds are very potent in retaining applied cells, with bone marrow-derived MSCs (bMSCs) having superior adhesion and proliferation potential on DBM when compared to normal human tendon (12).

Despite these potential benefits, there continues to be a lack of knowledge regarding DBM properties when using other cell sources (12). Thus, the purpose of this study was to evaluate the cellular adhesion and proliferation potential of concentrated bone marrow aspirate (cBMA), subacromial bursa-derived cells (SBDCs), tenocytes, and osteoblasts on a DBM scaffold. The authors hypothesized that there would be no significant difference in adhesion and proliferation potential between cell entities.

\section{MATERIALS AND METHODS SAMPLE HARVEST}

\section{Concentrated Bone Marrow Aspirate (cBMA)}

Bone marrow was aspirated from the proximal humerus of a healthy, 47-year-old, male patient during arthroscopic rotator cuff surgery, according to a previously published method (8). Following diagnostic arthroscopy, an 11-gauge trocar (Arthrex, Naples, FL, USA) was fit with a $60-\mathrm{ml}$ syringe and was inserted 2.5 to $3 \mathrm{~cm}$ into the medial aspect of the greater tuberosity (Bone Marrow Aspiration Kit, Arthrex, Naples, FL, USA). The tunnel created was later used to insert the first medial suture anchor. Prior to aspiration, the trocar was flushed 3 times with a heparin/saline solution containing $1,000 \mathrm{IU} / \mathrm{ml}$. For each shoulder aspiration, six 60-ml luer lock syringes were used, each of which was flushed 3 times with Anticoagulant Citrate Dextrose
Solution A (ACD-A) (Baxter Healthcare Corp) and had $2 \mathrm{cc}$ of the ACD-A solution. After attaching the $60-\mathrm{mL}$ syringe containing $2 \mathrm{cc}$ of ACD-A solution to the trocar, the syringe was pulled back to maximize suction. This standardized aspiration method was repeated six times allowing $18 \mathrm{ml}$ of bone marrow aspirate to flow into each of the six 60- $\mathrm{ml}$ syringes for a total of $120 \mathrm{ml}$ of bone marrow aspirate (BMA). This method of using multiple syringes was performed in order to prevent an excessive ratio of anticoagulant, in case $60 \mathrm{ml}$ of BMA was unable to be obtained in each syringe.

The BMA was transferred to a light absorption sensor technology system (Angel System, Arthrex, Naples, FL, USA) and concentrated using a $15 \%$ hematocrit setting (cBMA) (17). This setting was chosen based on preliminary data showing no significant changes in cellular yield, when using a higher concentration setting. This resulted in $\sim 3-4 \mathrm{cc}$ solution, rich in nucleated and concentrated bone marrow stromal cells, which was brought to the laboratory for cellular counting using the Z1 Coulter Counter (Beckman Coulter Life Sciences, Indianapolis, IN, USA). In order to obtain the total nucleated cell count, $0.1 \mathrm{cc}$ of cBMA was diluted in $9.9 \mathrm{cc}$ of saline. The number of nucleated cells in this 10 cc of solution was calculated and multiplied by 10 to obtain the total number of nucleated cells in $1 \mathrm{cc}$ of cBMA.

\section{Subacromial bursa-derived cells (SBDCs) and tenocytes}

Subacromial bursa and rotator cuff tendon were each obtained from the same healthy, 47-year-old, male patient using a laparoscopic grasper device and placed in sterile cups filled with saline. Samples were transferred to a tube containing $30 \mathrm{~mL}$ Collagenase P (Sigma-Aldrich, St. Louis, $\mathrm{MO}$, USA) reconstituted to $2 \mathrm{mg} / \mathrm{mL}$ with non-complete DMEM to avoid inactivation of the enzyme by fetal bovine serum (FBS). The tubes were then placed in a humified $37{ }^{\circ} \mathrm{C}$ rocking incubator at $5 \% \mathrm{CO}_{2}$ concentration for $2-3$ hours or until the tissue was visibly digested, followed by centrifugation at 2,000 RPM for 5 minutes to isolate the released cells. The supernatant was carefully aspirated, while preserving the cellular pellet. Cellular pellets were re-suspended and plated in $100 \mathrm{~mm}$ Primaria culture dishes (Fischer Scientific, Pittsburgh, PA, USA) with $10 \mathrm{~mL}$ complete Dulbecco's modified Eagle's medium (DMEM (1X), (ThermoFisher Scientific, Waltham, MA), containing $10 \%$ fetal bovine serum (FBS, (ThermoFisher) and $1 \%$ penicillin/streptomycin (Pen Strep Glutamine (100X), (ThermoFisher). Plates were incubated at $37^{\circ} \mathrm{C}$ with $5 \%$ $\mathrm{CO} 2$ and cells were grown to confluence with the complete media being changed every 3 to 4 days. 


\section{Osteoblasts}

Human osteoblasts (HOBs) were collected from femoral condyles of a healthy, 57-year-old, male patient during surgery. Samples were immediately cleaned of connective tissue and bone chips, minced with a bone reamer and scissors, roughly 5-7 $\mathrm{mm}$ in diameter, were cultured in Dulbecco's Modified Eagles Medium (DMEM) / Hams' F-12 media containing 10\% fetal bovine serum (FBS) (Thermo Scientific) and $100 \mathrm{U} / \mathrm{ml}$ penicillin, and $100 \mu \mathrm{g} / \mathrm{ml}$ penicillin streptomycin (PS) (Thermo Scientific). After 2 weeks bone chips were removed and cells were grown until confluent. Assessment of alkaline phosphatase activity and gene expression demonstrated highly consistent results as previously shown by Zhang et. al. (data not shown) (18).

\section{Cell counting}

Culture dishes for osteoblasts, tenocytes and bursa cells were aspirated of media and incubated at $37{ }^{\circ} \mathrm{C}$ with 1.5 $\mathrm{mL}$ sterile trypsin in EDTA for 20 minutes. After incubation, $1.5 \mathrm{~mL}$ of complete DMEM was added to the dish to inactivate the trypsin. Two transparent cuvettes were filled with $9.9 \mathrm{~mL}$ of $0.9 \% \mathrm{NaCl}$ for each culture dish that was to be counted. A $100 \mu \mathrm{L}$ sample of the cellular solution was added into both cuvettes. Samples were counted using a Z1 Coulter Particle Counter (Beckman Coulter Life Sciences, Indianapolis, IN, USA). Prior to sample counting, the machine was flushed twice with a cuvette containing $0.9 \% \mathrm{NaCl}$. A count of this cuvette was also made to serve as control. Each of the two samples per dish were then analyzed to determine the number of cells per $\mathrm{mL}$ of sample. The control count was subtracted from each sample count and the mean of both adjusted counts was calculated.

\section{CONFIRMATION OF MSCS}

In order to confirm the presence of mesenchymal stem cells, cellular adhesion to tissue culture plastic with the ability for form colonies and flow cytometry was performed to identify specific MSC markers for the cBMA and SBDCs. One million passage- 3 cells were resuspended in $500 \mu \mathrm{L}$ of PBS containing a fluorescein isothiocyanate- (FITC-) or phycoerythrin(PE-) antibody. The FITC- or PE antibodies against surface markers CD105 and CD90 (Ancell, Bayport, MN), along with CD73, CD45, and CD31 (BD Biosciences, San Diego, CA) were used. The presence of CD105, CD90, CD73, along with the absence of CD45 and CD31 were used to determine the criteria for mesenchymal stem cells (19). Cell fluorescence was evaluated by flow cytometry using an LSRII (BD Biosciences, San Diego, CA), and data were analyzed using a BD FACS Diva Software (BD Biosciences, San Diego, CA) (20).

\section{EXPERIMENTAL DESIGN}

The study meets the ethical standards of the journal (21). One 5 x $5 \mathrm{~mm}$ square of DBM (AlloSync ${ }^{\mathrm{TM}}$, Arthrex Inc., Naples, FL) was placed into each well of a 24-well tissue culture plate (BD Laboratories, Franklin Lakes, NJ), and subacromial bursa-derived cells, tenocytes, and osteoblasts were trypsinized and counted as described above. Cells were plated onto each sample at a concentration of 40,000 cell/scaffold sample in a $100 \mu \mathrm{L}$ volume of complete media. A $100 \mu \mathrm{L}$ volume of pure cBMA was applied without prior need of culture, in order to keep the process as clinically applicable as possible. For all experiments, a period of 60 minutes was allowed prior to media being added to ensure adhesion, as most cells adhere to substrate through integrin receptors in less than 30 minutes (12).

As cBMA is still the most common source for biologic augmentation in the setting of arthroscopic shoulder surgery (2), only that sample was further investigated with regards to adhesion and proliferation potential on the scaffold in an arthroscopic simulation. Besides, the authors were concerned about the adhesion potential of cBMA, as the soaked scaffold changes colour from red to white during arthroscopic surgery, leaving unknown, as to whether the cells are still adhered when the rotator cuff is finally repaired. In order to mimic these intraoperative arthroscopic conditions, samples undergoing arthroscopic simulation were taken out of their respective wells, placed into a 100 $\mathrm{ml}$ bottle of sterile saline connected to an arthroscopic simulation apparatus (12). The bottle, in which the DBM was placed, simulated a joint during arthroscopic surgery. Inflow and outflow tubes were continuously pumping saline at a pressure of $40 \mathrm{~mm} \mathrm{Hg}$ via an arthroscopic pump (Continuous Wave II Arthroscopy Pump, Arthrex) for a length of 60 minutes (12). A period of 60 minutes was chosen based on an approximated maximum time the cell containing scaffold is expected to be in an arthroscopic environment during surgery. Samples not receiving arthroscopic simulation were incubated in their respective wells for 60 minutes. All samples were then placed into individual wells and incubated to assess for cellular adhesion and proliferation potential (12).

\section{CELLULAR ADHESION ASSAY}

Samples were incubated for 24 hours, removed from the wells, placed into a clean well, and washed 3 times with $1 \mathrm{x}$ phosphate buffered saline to remove non-adherent cells. This ensured that only cells with the ability to attach to the scaffold would be counted. Adherent cells were released from the scaffold using $0.5 \mathrm{ml} /$ well of $0.5 \%$ trypsin/ ethylenediamine tetra-acetic acid. The cells were diluted in 
9,5 ml's of saline, and each well was counted 3 times using the Coulter Counter for accuracy (12).

\section{CELLULAR PROLIFERATION ASSAY}

To determine cellular growth, a cellular proliferation XTT assay was performed after 48 hours in culture (22). After culturing, the wells were aspirated, and the DBM samples were moved to a new 24 -well plate. $600 \mu \mathrm{L}$ of complete medium and $300 \mu \mathrm{L}(0.3 \mathrm{mg} / \mathrm{mL})$ of XTT labeling mixture $(0.1$ $\mathrm{ml}$ electron coupling reagent/ $5 \mathrm{~mL}$ XTT labeling reagent) was added to each well and allowed to incubate overnight at $37^{\circ} \mathrm{C}$. After incubating, the labeling mixture was removed and DBM smaples were transferred to new wells. Absorbance was read at $450 \mathrm{~nm}$ with a reference wavelength of $650 \mathrm{~nm}$. This assay forms a formazan dye through the cleavage of the terazolium salt XTT by active cells, therefore, the amount of dye detected is a direct correlation to the number of active cells in the culture. Each treatment group consisted of six replicates.

\section{HISTOLOGY}

To histologically evaluate the presence of attached and proliferated cells, scaffold samples treated with and without cBMA were placed in a histology cassette. The cells were fixed in $2 \%$ paraformaldehyde in phosphate buffered saline (PBS) and refrigerated at $4^{\circ} \mathrm{C}$ for a minimum of 24 hours. After fixation, the samples were dehydrated stepwise with increasing concentrations of ethanol and cleared in xylene. The samples were then embedded in paraffin. $5 \mu \mathrm{m}$ sections were taken with a microtome and stained with hematoxylin and eosin. Each slide was microscopically analyzed at $4 \mathrm{X}$ and $10 \mathrm{X}$ magnification.

\section{ELECTRON MICROSCOPY}

Scanning electron microscopy (SEM) using a Philips 515 scanning electron microscope (Philips Nederland BV, Eindhoven, Netherlands) was employed to help visualize the porosity and overall structure of each scaffold along with the presence of adhered and proliferated cBMA cells.

\section{STATISTICAL ANALYSIS}

Data was summarized with mean and standard deviation (SD) as well as median and interquartile range (IQR). Given the small sample size, non-parametric inferential methods were used. Differences in adhesion and proliferation potential between the cell lines (subacromial bursa, osteoblasts, tenocytes, and concentrated bone marrow aspirate) were compared with the Kruskal Wallis test. If the Kruskal Wallis test was statistically significant, pairwise comparisons were carried out with the Wilcoxon rank-sum test. Difference in adhesion and proliferation potential between the wash-out and no-wash-out states in the cBMA group were compared using the Wilcoxon sign-rank test. A p-value of less than 0.05 was considered statistically significant. All statistical analyses were performed using Stata (StataCorp 2017. Stata Statistical Software: Release 15. College Station, TX: StataCorp LLC).

\section{RESULTS}

\section{Nucleated Cell Count and Confirmation of MSCs}

Concentrated bone marrow aspirate was immediately counted in the laboratory prior to plating and revealed a concentration of 402,000 + 20,400 nucleated cells $/ \mathrm{ml}$. Therefore, a $100 \mathrm{ul}$ aliquot of cBMA when delived to the DBM had 40,000 nucleated cells in it.

Cells derived from cBMA and digested bursa (SBDCs) were cultured for flow cytometry analysis. The results revealed the presence of surface cell markers CD90 (100\% positive and $95 \%$ positive), CD105 (96.5\% positive and $95.5 \%$ positive) and CD73 (90\% positive and $91 \%$ positive) for cBMA and SADC, respectively (figure 1). All samples were negative for surface markers CD45 and CD31. Further, when plated onto tissue culture plastic (TCP), both cBMA and SBDCs attached to TCP and had the ability to form colonies.

\section{Cellular Adhesion Potential on Scaffolds}

There was no significant difference in cellular adhesion potential (total number of adhered cells) on DBM scaffolds ( $\mathrm{p}=0.168$; figure 2 ) when comparing subacromial bursa-derived cells (mean \pm SD: 25,775 $\pm 11,351$; median: 25,750; IQR: 14,150), osteoblasts (mean \pm SD: 33,113 \pm 2,709; median: 33,025; IQR: 4,275), tenocytes (mean \pm SD: 25,000 \pm 3,864; median: 26,150; IQR: 5,550), and cBMA (mean \pm SD: 25,025 \pm 6,206; median: 23,900; IQR: 8,450).

\section{Cellular Proliferation Potential on Scaffolds}

There was a significant difference in cellular proliferation potential (corrected absorbance) on DBM scaffolds between cell entities ( $\mathrm{p}=0.027$; figure 3 ). Osteoblasts (mean \pm SD: $0.279 \pm 0.009$; median: 0.276; IQR: 0.011 ) showed a significantly higher proliferation potential compared to subacromial bursa-derived cells (mean \pm SD: $0.248 \pm 0.015$; median: 0.247; IQR: 0.021; $\mathrm{p}=0.021$ ), tenocytes (mean $\pm \mathrm{SD}: 0.239 \pm 0.011$; median: 0.236; 

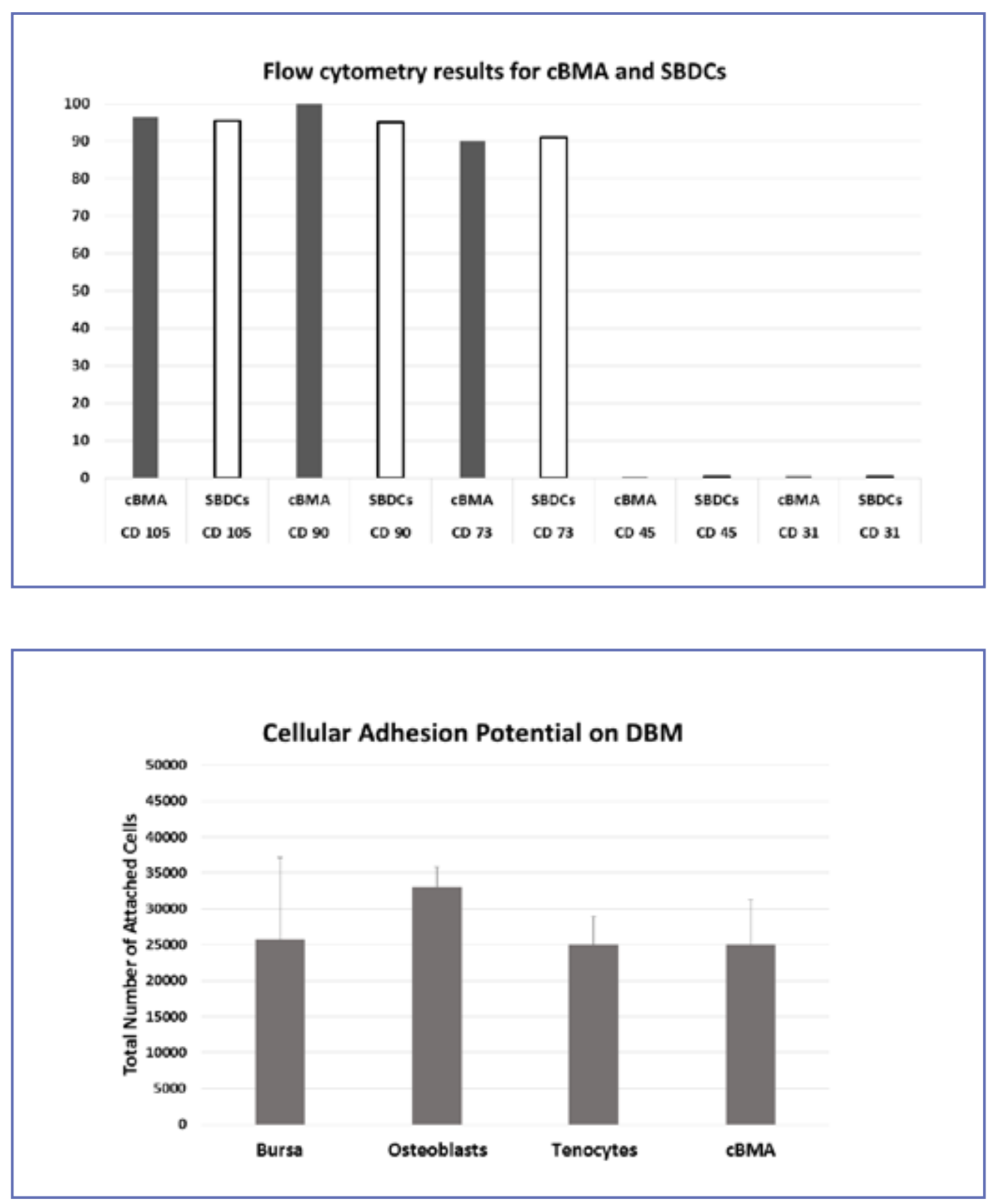

Figure 1. Confirmation of MSC specific surface markers using FACS analysis. Abbreviations: $\mathrm{cBMA}=$ concentrated bone marrow aspirate; SBDCs = subacromial bursa-derived cells; $M S C=$ mesenchymal stem cell; $F A C S=$ fluorescence-activated cell sorting.
Figure 2. Demonstrating cellular adhesion potential (total number of adhered cells) of various cell entities on DBM scaffold (abbreviations: $\mathrm{DBM}=$ demineralized bone matrix; $\mathrm{cBMA}=$ concentrated bone marrow aspirate).
IQR: 0.017; $\mathrm{p}=0.021$ ), and cBMA (mean \pm SD: $0.247 \pm$ 0.007; median: 0.245; IQR: 0.011; $\mathrm{p}=0.021$ ). No significant difference was found in cellular proliferation when comparing subacromial bursa-derived cells, tenocytes, and cBMA ( $p>0.05$, respectively).

\section{Arthroscopic Simulation}

Arthroscopic simulation did not significantly alter the level of cellular adhesion ( $\mathrm{p}=0.715$; figure 4$)$ or cellular prolifer- ation ( $\mathrm{p}=0.947$; figure 5) when investigating DBM samples augmented with cBMA.

\section{Histology and Electron Microscopy}

Histology sections confirmed the presence of many attached and proliferated cells within the matrix of scaffolds augmented with cBMA (figure 6). Scanning electron microscopy further allowed for visualizing the clear differences in matrix architecture of scaffolds augmented with 

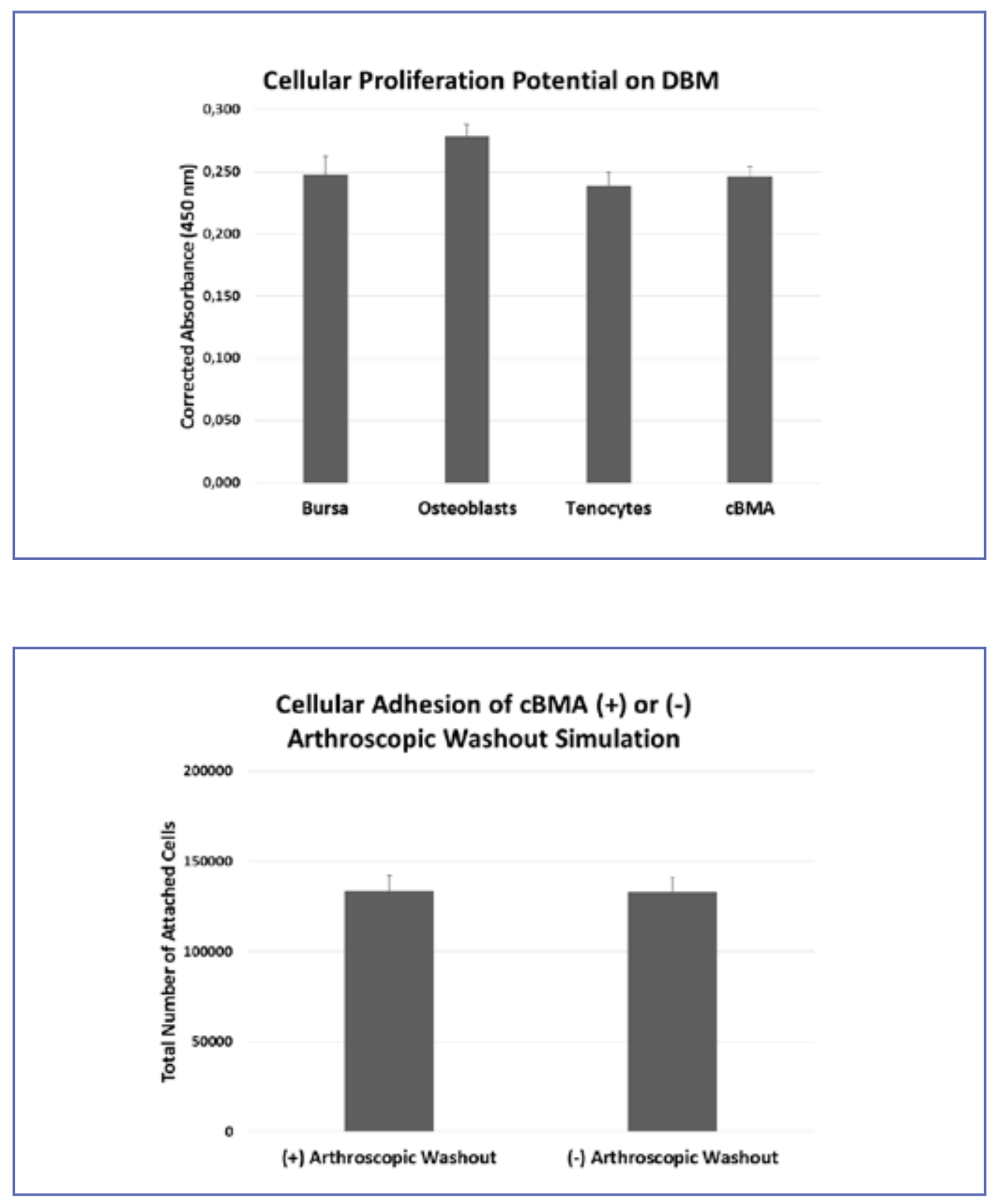

Figure 3. Demonstrating cellular proliferation potential (corrected absorbance) of various cell entities on DBM scaffold. Abbreviations: DBM = demineralized bone matrix; $\mathrm{CBMA}=$ concentrated bone marrow aspirate.
Figure 4. Demonstrating cellular adhesion potential of cBMA on DBM scaffolds with and without arthroscopic wash-out simulation. Abbreviations: $\mathrm{DBM}=$ demineralized bone matrix; $c B M A=$ concentrated bone marrow aspirate.
cBMA, underlining the high adhesion and proliferation potential of cBMA to the DBM scaffold (figure 7).

\section{DISCUSSION}

The most important finding of the study was that subacromial bursa-derived cells, osteoblasts, tenocytes, and cBMA demonstrated high cellular adhesion and proliferation potential on DBM scaffolds. Arthroscopic washout did not result in a significant decrease in adhesion or proliferation in DBM scaffolds treated with cBMA. Osteoblasts showed a significantly higher proliferation potential when compared to the other cell entities. These findings highlight the ability of DBM scaffolds to sufficiently deliver and retain applied cells of various origins at a targeted zone as well as their potency as biologic augments in the setting of arthroscopic rotator cuff repair.

DBM scaffolds demonstrate high biocompatibility and osteoinductive potential, thus their use as biologic augments in the field of tendon repair has become more popular (12-16). 


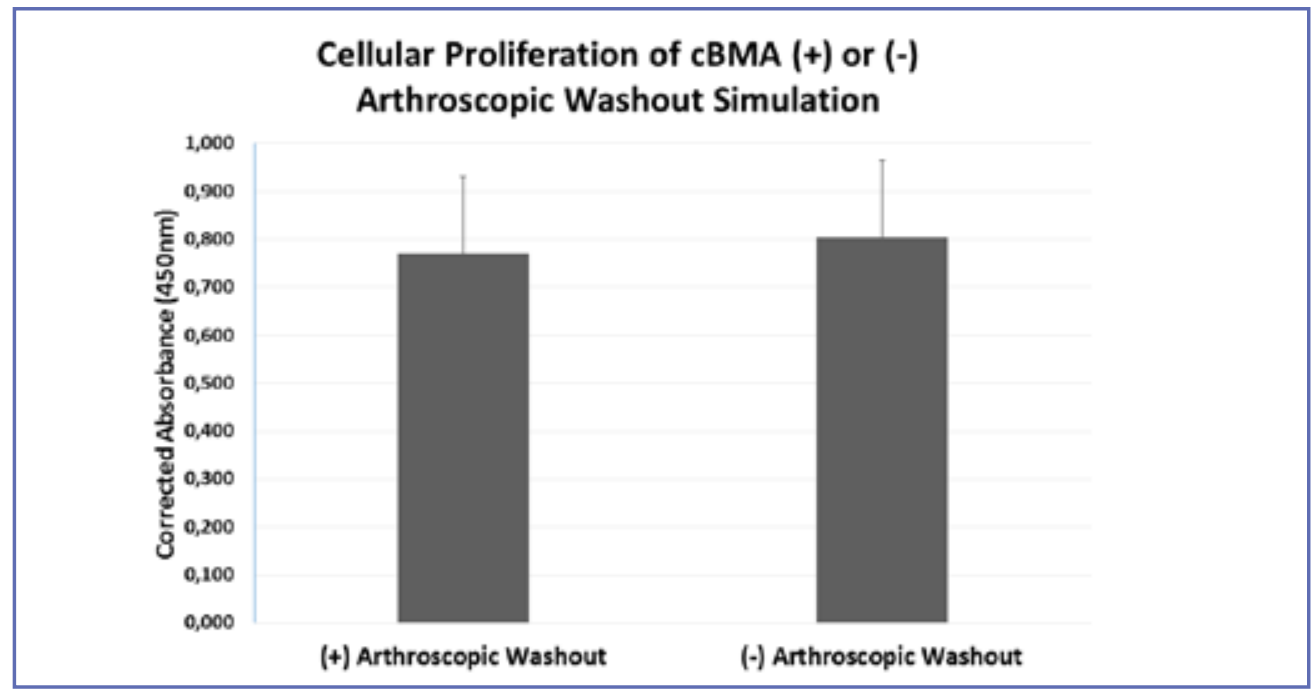

Figure 5. Demonstrating cellular proliferation potential of cBMA on DBM scaffolds with and without arthroscopic wash-out simulation. Abbreviations: $\mathrm{DBM}=$ demineralized bone matrix; $c B M A=$ concentrated bone marrow aspirate.

Figure 6. Confirmation of many attached cells (orange circle) on representative histology section of DBM scaffold before (A) and after (B) application of cBMA (4X magnification).

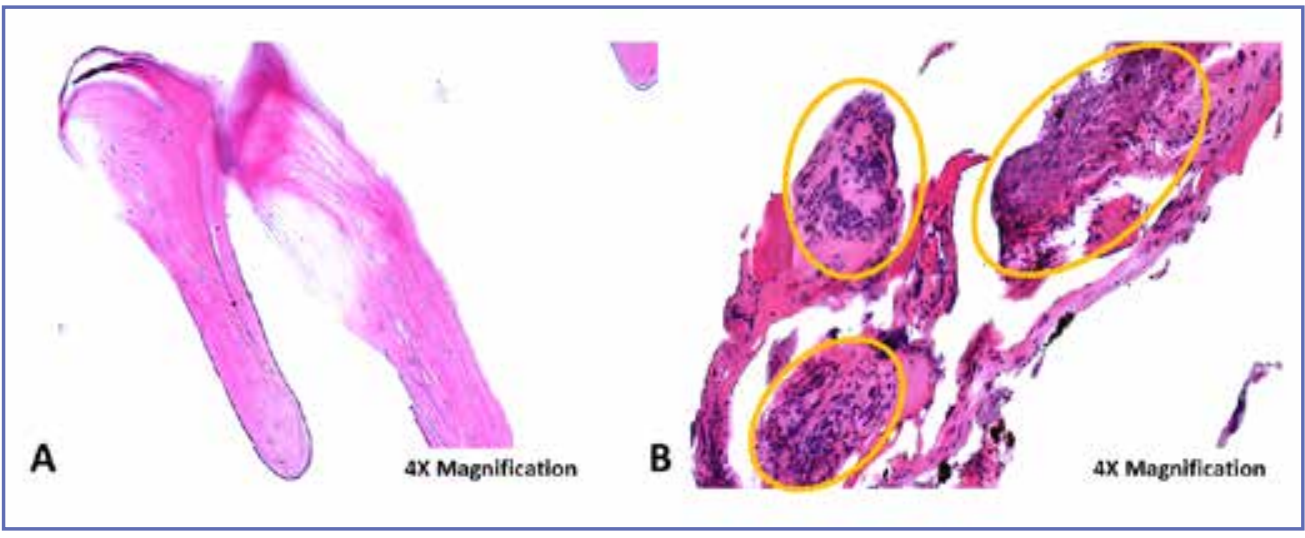

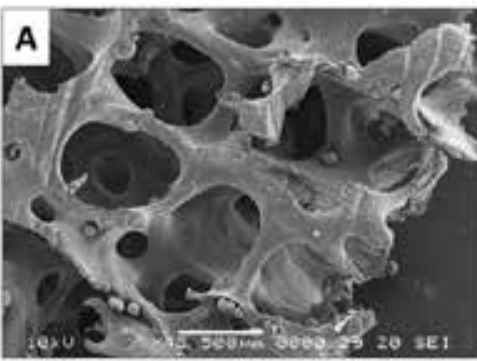
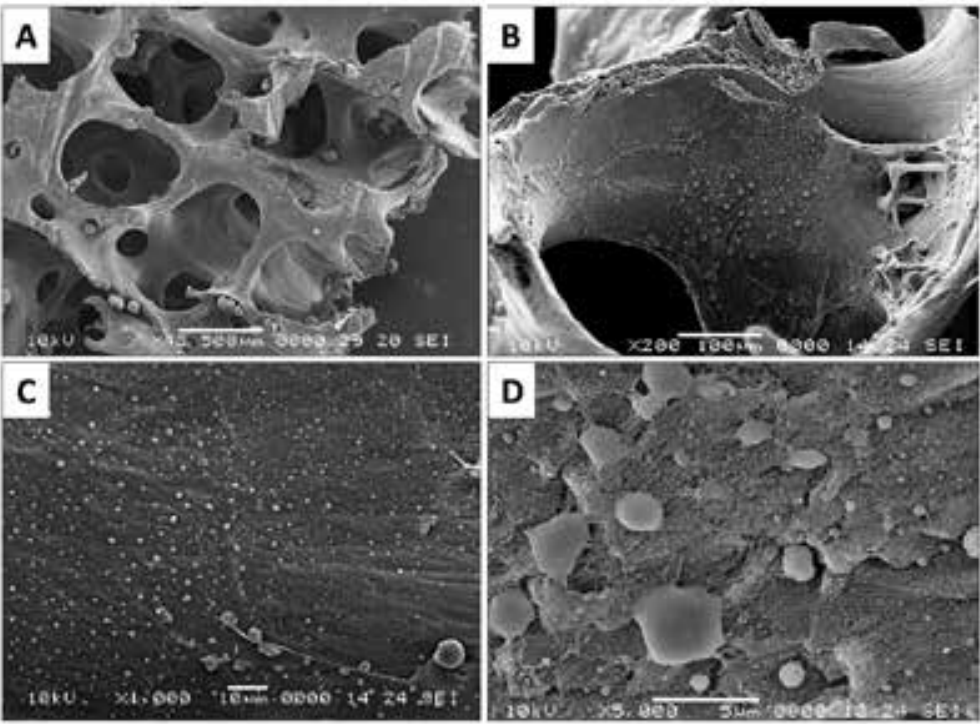

Figure 7. Integration of cells in scaffold matrix visualized using scanning electron microscopy at (A) 43X, (B) 200X, (C) 1000X, and (D) 5000X magnification. 
Recent studies have focused on investigating the interaction as well as combination of these scaffolds with additional biologic adjuvants, in order to maximize healing potential (12). As adhesion and proliferation of applied cells depends on the physical architecture of the material, the interaction of transmembrane receptors with environmental substrates along with conductive growth factor expression are essential. DBM scaffolds have undergone further development to improve these properties $(23,24)$, especially with regards to greater surface area and composition of highly porous material to enhance cellular adhesion and proliferation $(12,25)$. This is consistent with the results of the scanning electron microscopy of the present study.

However, previous studies only investigated the cellular adhesion and proliferation of bMSCs on DBM scaffolds, leaving a lack of knowledge regarding the interaction with other cell entities. Thus, the present study compared various cell entities, showing that DBM scaffolds were able to reliably retain applied SBDCs, cBMA, human osteoblasts, and tenocytes. This may be of clinical importance, in order to maximize the effect of applied biologic adjuvants and to create an ideal healing environment, especially in the setting of massive or revision rotator cuff tears $(3,26)$. Although there was no significant difference in cellular adhesion potential, osteoblasts demonstrated a significantly higher proliferation potential compared to all other cell entities. This may be a result of the high osteoinductive potential of these scaffolds and release of bone morphogenetic proteins, justifying their clinical use in fracture non-union or spinal fusion for augmentation of bone formation (14). Besides, It has to be taken into account that osteoblasts were not harvested from the same patient as SBDCs, cBMA, and tenocytes.

In the setting of arthroscopic rotator cuff repair, application of cBMA remains the "gold standard" for biologic augmentation, with previous literature suggesting significant improvement in healing outcomes with its use (2-4). Thus, our results, including the high in vitro adhesion and proliferation potential of cBMA on DBM scaffolds, seem promising for its clinical application, especially along with the histologic and electron microscopic evaluation showing sufficient integration of the applied cells in the scaffold matrix. Additionally, no decrease in cellular measures were observed after a simulated arthroscopic washout. This is of importance, as rotator cuff repair is commonly performed arthroscopically, requiring the cells to adhere and proliferate while resisting arthroscopic fluid pressure.

Besides, recent studies suggest subacromial bursa to be a potential, easily accessible source for MSCs that can be used for biologic augmentation of rotator cuff repairs, as they fulfil all required characteristics including multilineage differentiation (9-11). Moreover, Dyrna et al. found that human SBDCs demonstrated superior tissue engraftment and survival in an animal tendon model when compared to bMSCs (27). The present study found that SBDCs also adhere and proliferate on DBM scaffolds, highlighting their potential as an easily accessible biologic augment in combination with scaffolds to keep the cells at the targeted zone. However, further research needs to be done to confirm retainment of these cells in a clinical setting.

There were several limitations to the study. This is an in vitro study without direct evidence of the effects of these implanted cells. SBDCs, cBMA, tenocytes and osteoblasts were only obtained from a single donor, in order to control for the wide variability. However, this study design is not able to reflect the existing large inter-individual variability of biologic adjuvants. In addition, SBDCs, cBMA and tenocytes were harvested from the same patient, whereas the osteoblasts were obtained from another patient. This may have influenced the comparison of cellular measures. Additionally, only one scaffold type was used to compare the cellular adhesion and proliferation potential of the different cell entities. Another limitation was the simulated arthroscopy for certain samples. While this study allowed a method to reliably reproduce the conditions of constant water pressure that is seen intra-operatively, the model may not allow for the in vivo surgical setting. For instance, our model was not able to mimic flow turbulences which may be generated due to changes in flow or the use of arthroscopic surgical tools. However, the scaffolds and cells were exposed to these conditions for an extended amount of time (60 minutes), in order to account for variability of operating time.

In conclusion, subacromial bursa-derived cells and concentrated bone marrow aspirate demonstrated high cellular adhesion and proliferation potential on demineralized bone matrix scaffolds, without a significant decrease after arthroscopic wash-out simulation. This may correlate to improved patient reported outcome with increased delivery of mesenchymal stem cells during arthroscopic rotator cuff repair.

\section{CONFLICT OF INTERESTS}

The authors declare that there are no conflict of interests

\section{REFERENCES}

1. Zumstein MA, Ladermann A, Raniga S, Schar MO. The biology of rotator cuff healing. Orthop Traumatol Surg Res. 2017;103:S1-S10.

2. Imam MA, Holton J, Horriat $S$, et al. A systematic review of the concept and clinical applications of bone marrow aspirate concentrate in tendon pathology. SICOT J. 2017;3:58. 
3. Hernigou P, Flouzat Lachaniette CH, Delambre J, et al. Biologic augmentation of rotator cuff repair with mesenchymal stem cells during arthroscopy improves healing and prevents further tears: a case-controlled study. Int Orthop. 2014;38:1811-1818.

4. Ellera Gomes JL, da Silva RC, Silla LM, Abreu MR, Pellanda R. Conventional rotator cuff repair complemented by the aid of mononuclear autologous stem cells. Knee Surg Sports Traumatol Arthrosc. 2012;20:373-377.

5. Berebichez-Fridman R, Gomez-Garcia R, Granados-Montiel J, et al. The Holy Grail of Orthopedic Surgery: Mesenchymal Stem Cells-Their Current Uses and Potential Applications. Stem Cells Int. 2017;2017:2638305.

6. Via AG, Frizziero A, Oliva F. Biological properties of mesenchymal Stem Cells from different sources. Muscles Ligaments Tendons J. 2012;16:154-162.

7. Giai Via A, McCarthy MB, de Girolamo L, Ragni E, Oliva F, Maffulli N. Making Them Commit: Strategies to Influence Phenotypic Differentiation in Mesenchymal Stem Cells. Sports Med Artbrosc Rev. 2018;26:64-69.

8. Mazzocca AD, McCarthy MB, Chowaniec DM, Cote MP, Arciero RA, Drissi H. Rapid isolation of human stem cells (connective tissue progenitor cells) from the proximal humerus during arthroscopic rotator cuff surgery. Am J Sports Med. 2010;38:1438-1447.

9. Steinert AF, Kunz M, Prager P, et al. Characterization of bursa subacromialis-derived mesenchymal stem cells. Stem Cell Res Ther. 2015;6:114.

10. Song N, Armstrong AD, Li F, Ouyang H, Niyibizi C. Multipotent mesenchymal stem cells from human subacromial bursa: potential for cell based tendon tissue engineering. Tissue Eng Part A. 2014;20:239-249.

11. Utsunomiya H, Uchida S, Sekiya I, Sakai A, Moridera K, Nakamura T. Isolation and characterization of human mesenchymal stem cells derived from shoulder tissues involved in rotator cuff tears. Am J Sports Med. 2013;41:657-668.

12. Hoberman AR, Cirino C, McCarthy MB, et al. Bone Marrow-Derived Mesenchymal Stromal Cells Enhanced by Platelet-Rich Plasma Maintain Adhesion to Scaffolds in Arthroscopic Simulation. Arthroscopy. 2018;34:872-881.

13. Shahmoradi SR, Kabir Salmani M, Soleimanpour HR, et al. Induction of Chondrogenic Differentiation in Human Mesenchymal Stem Cells Cultured on Human Demineralized Bone Matrix Scaffold under Hydrostatic Pressure. Tissue Eng Regen Med. 2019;16:69-80.

14. Sundar S, Pendegrass CJ, Blunn GW. Tendon bone healing can be enhanced by demineralized bone matrix: a functional and histological study. J Biomed Mater Res B Appl Biomater. 2009;88:115-122.

15. Thangarajah T, Sanghani-Kerai A, Henshaw F, Lambert SM, Pendegrass C, Blunn G. Application of a Demineralized Corti- cal Bone Matrix and Bone Marrow-Derived Mesenchymal Stem Cells in a Model of Chronic Rotator Cuff Degeneration. Am J Sports Med. 2018;46:98-108.

16. Hexter AT, Pendegrass C, Haddad F, Blunn G. Demineralized Bone Matrix to Augment Tendon-Bone Healing: A Systematic Review. Orthop J Sports Med. 2017;5:2325967117734517.

17. Voss A, McCarthy MB, Singh H, et al. The Influence of Trocar Fenestration and Volume on Connective Tissue Progenitor Cells (Stem Cells) in Arthroscopic Bone Marrow Aspiration From the Proximal Humerus. Arthroscopy. 2017;33:1167-1174 e1161.

18. Zhang H, Lewis CG, Aronow MS, Gronowicz GA. The effects of patient age on human osteoblasts' response to Ti-6Al-4V implants in vitro. J Orthop Res. 2004;22:30-38.

19. Dominici M, Le Blanc K, Mueller I, et al. Minimal criteria for defining multipotent mesenchymal stromal cells. The International Society for Cellular Therapy position statement. Cytotherapy. 2006;8:315-317.

20. Katagiri K, Matsukura Y, Muneta T, et al. Fibrous Synovium Releases Higher Numbers of Mesenchymal Stem Cells Than Adipose Synovium in a Suspended Synovium Culture Model. Arthroscopy. 2017;33:800-810.

21. Padulo J, Oliva F, Frizziero A, Maffulli N. Muscles, Ligaments and Tendons Journal - Basic principles and recommendations in clinical and field Science Research: 2016 Update. Muscles Ligaments Tendons J. 2016;6:1-5.

22. Roehm NW, Rodgers GH, Hatfield SM, Glasebrook AL. An improved colorimetric assay for cell proliferation and viability utilizing the tetrazolium salt XTT. I Immunol Methods. 1991;142:257-265.

23. Hidalgo-Bastida LA, Cartmell SH. Mesenchymal stem cells, osteoblasts and extracellular matrix proteins: enhancing cell adhesion and differentiation for bone tissue engineering. Tissue Eng Part B Rev. 2010;16:405-412.

24. Derwin KA, Badylak SF, Steinmann SP, Iannotti JP. Extracellular matrix scaffold devices for rotator cuff repair. J Shoulder Elbow Surg. 2010;19:467-476.

25. Murphy CM, Haugh MG, O’Brien FJ. The effect of mean pore size on cell attachment, proliferation and migration in collagen-glycosaminoglycan scaffolds for bone tissue engineering. Biomaterials. 2010;31:461-466.

26. Nho SJ, Delos D, Yadav H, et al. Biomechanical and biologic augmentation for the treatment of massive rotator cuff tears. Am J Sports Med. 2010;38:619-629.

27. Dyrna F, Zakko P, Pauzenberger L, McCarthy MB, Mazzocca AD, Dyment NA. Human Subacromial Bursal Cells Display Superior Engraftment Versus Bone Marrow Stromal Cells in Murine Tendon Repair. Am J Sports Med. 2018;46:3511-3520. 